\title{
Preventive effect of Elateriospermum tapos seed extract against obese Sprague Dawley rats
}

\begin{abstract}
This study aimed to investigate the preventive role of Elateriospermum tapos seed extract against obese Sprague Dawley rats through assessment of bodyweight, caloric intake, organs weight, biological assays and histopathology. Thirty-six male Sprague Dawley rats were assigned into six groups of normal control (G1) group fed with standard chow diet, negative control (G2), positive control (G3) and treatment groups (G4, G5 and G6) were on high-fat and cafeteria diet for 9 weeks. G3 group was given $10 \mathrm{mg} \mathrm{kg}-1$ of Orlistat while treatment groups were supplemented with E. tapos seed extract of $5 \mathrm{mg} \mathrm{kg}-1,25 \mathrm{mg} \mathrm{kg}-1$ and $125 \mathrm{mg} \mathrm{kg}-1$ orally daily for another 10 weeks. Bodyweight and food intake were monitored weekly. At the end, liver, retroperitoneal white adipose tissue (rpWAT) and blood were collected for analysis of total cholesterol (TC), triglycerides (TG), low-density (LDL-C) and high density lipoprotein (HDL-C). The E. tapos seed treated groups showed significant $(\mathrm{p}<0.05)$ reduction in bodyweight, caloric intake, liver and rpWAT weight as compared to the G2 group. G6 group showed tremendous improvement of liver histopathology and biological assay. There was a significant decrease $(p<0.05)$ of TC, TG, and LDL-C level and significant increase $(p<0.05)$ of HDL-C in the E. tapos seed treated group as compared to G2 group. Based on the findings, E. tapos seed extract exhibited a great potential as an anti-obesity. The extract promoted the fat oxidation by removing the uptake and storage of fat by the adipose cells and also decrease the fatty acid synthesis.
\end{abstract}

Keyword: Cafeteria diet; Elateriospermum tapos; High-fat diet; Histology; Lipid profile; Obesity 